\title{
Symptomatic relapse of HIV-associated cryptococcal meningitis in South Africa: The role of inadequate secondary prophylaxis
}

\author{
Joseph N Jarvis, Graeme Meintjes, Zomzi Williams, Kevin Rebe, Thomas S Harrison
}

Objectives. Cryptococcal meningitis is the most common cause of adult meningitis in southern Africa. Much of this disease burden is thought to be due to symptomatic relapse of previously treated infection. We studied the contribution of inadequate secondary fluconazole prophylaxis to symptomatic relapses of cryptococcal meningitis.

Design. A prospective observational study of patients presenting with laboratory-confirmed symptomatic relapse of HIV-associated cryptococcal meningitis between January 2007 and December 2008 at GF Jooste Hospital, a public sector adult referral hospital in Cape Town.

Outcome measures. Relapse episodes were categorised into: (i) patients not taking fluconazole prophylaxis; (ii) immune reconstitution inflammatory syndrome (IRIS); and (iii) relapses occurring prior to ART in patients taking fluconazole. In-hospital mortality was recorded.

Results. There were 69 relapse episodes, accounting for $23 \%$ of all cases of cryptococcal meningitis; $43 \%(N=30)$ of relapse episodes were in patients not receiving fluconazole prophylaxis, $45 \%(N=31)$ were due to IRIS, and $12 \%(N=8)$ were in patients pre-ART taking fluconazole. Patients developing relapse due to inadequate secondary prophylaxis had severe disease and high in-hospital mortality $(33 \%)$. Of the 30 patients not taking fluconazole, $47 \%(N=14)$ had not been prescribed secondary prophylaxis by their health care providers. We documented no relapses due to fluconazole resistance in these patients who received amphotericin B as initial therapy.

Conclusions. A large number of relapses of cryptococcal meningitis are due to failed prescription, dispensing and referral for or adherence to secondary fluconazole prophylaxis. Interventions to improve the use of secondary fluconazole prophylaxis are essential.

S Afr Med J 2010; 100: 378-382.
Cryptococcal meningitis (CM) is now the most common cause of adult meningitis in southern Africa ${ }^{1,2}$ and is a major burden on local health care services. ${ }^{3}$ Treatment remains unsatisfactory, with acute mortality in Africa ranging from $30 \%$ to $50 \%$, even with optimal therapy., ${ }^{4,5}$ Consequently, CM is a major cause of mortality in African HIV-infected patients, accounting for 13 $44 \%$ of all deaths. ${ }^{6-8}$ Estimates from the Centers for Disease Control and Prevention (CDC) show that CM is responsible for 504000 deaths in sub-Saharan Africa annually. ${ }^{9}$

Infectious Diseases Unit, GF Jooste Hospital, Cape Town Joseph N Jarvis, MB BS, MRCP (UK)

Graeme Meintjes, MB ChB, MRCP (UK), FCP (SA)

Zomzi Williams, DipGenNurs, Dip PHC

Kevin Rebe, MB ChB, FCP (SA)

Division of Infectious Diseases and HIV Medicine, Department of Medicine, University of Cape Town

Joseph N Jarvis, MB BS, MRCP (UK)

Graeme Meintjes, MB ChB, MRCP (UK), FCP (SA)

Kevin Rebe, MB ChB, FCP (SA)

Centre for Infection, Department of Cellular and Molecular Medicine, St. George's University of London, UK

Joseph N Jarvis, MB BS, MRCP (UK)

Thomas S Harrison, MD, FRCP (UK)

Desmond Tutu HIV Centre, Institute of Infectious Disease and Molecular Medicine, University of Cape Town

Joseph N Jarvis, MB BS, MRCP (UK)

Institute of Infectious Disease and Molecular Medicine, University of Cape Town Graeme Meintjes, MB ChB, MRCP (UK), FCP (SA)
A sizeable proportion of the cryptococcal disease burden is thought to be due to symptomatic relapse of previously treated infection. Recent work has highlighted the contribution made by immune reconstitution syndrome (IRIS) and fluconazole resistance to relapse in South African patients taking secondary fluconazole prophylaxis. ${ }^{10-12}$

Without secondary prophylaxis, at least $50-60 \%$ of patients will have disease relapse. ${ }^{13-15}$ Properly used secondary fluconazole prophylaxis is highly effective, increasing recurrence-free survival by more than 13 times at 6 months. ${ }^{16,17}$ Fluconazole has been freely available in South Africa since 2001 via Pfizer's donation programme, and current South African guidelines for the treatment of HIV-associated CM recommend fluconazole consolidation treatment for all patients, following initial amphotericin B therapy, then secondary fluconazole prophylaxis either lifelong or until the CD4 count is $>200$ for 6 months on ART. ${ }^{18}$ However, evidence suggests that the use of secondary fluconazole prophylaxis in South Africa is poor, with only $31.5 \%$ of initial prescriptions re-filled at 2 months, and only $6 \%$ of patients returning for fluconazole at 1 year. ${ }^{19}$

To examine the contribution of inadequate fluconazole prophylaxis to symptomatic relapses of cryptococcal disease we carried out a prospective observational study at GF Jooste Hospital, a public sector adult referral hospital in Cape Town serving a population of 1.3 million (including a large part of Khayelitsha township, population 400 000, with HIV antenatal seroprevalence of $32.7 \%$ in 2006). ${ }^{20}$ Cryptococcus is the commonest cause of meningitis at the hospital, accounting for $63 \%$ of microbiologically confirmed cases, ${ }^{21}$ and the disease 
burden is high, with over 100 India ink-positive cases per year. $^{22}$

\section{Methods}

\section{Participants}

The study was approved by the Research Ethics Committee of the University of Cape Town. Between January 2007 and December 2008, all patients presenting to GF Jooste Hospital with a laboratory-confirmed diagnosis of cryptococcal meningitis were prospectively identified. Cases of symptomatic relapse were defined as occurring in patients with the following characteristics: (i) a previous laboratory-confirmed case of CM; (ii) recurrence of typical CM symptoms; (iii) CSF India ink, antigen test (CRAG) and/or culture positive for Cryptococcus neoformans at this presentation; and (iv) no alternative diagnosis.

A patient could be classified as having more than one relapse episode, provided that there had been a resolution of symptoms and an interval of at least 1 month between episodes. During the study period, the standard treatment of $\mathrm{CM}$ at the hospital consisted of 2 weeks of amphotericin $B$ at a dose of $1 \mathrm{mg} / \mathrm{kg}$, followed by fluconazole $400 \mathrm{mg}$ daily as consolidation treatment for 8 weeks, then long-term secondary prophylaxis with fluconazole $200 \mathrm{mg}$ daily, as per current guidelines. ${ }^{18}$ As part of ongoing clinical studies, 8 of our patients had also received additional induction treatment during their initial episode ( 2 received flucytosine, 4 received IFN $\gamma$ and flucytosine, and 2 received fluconazole).

\section{Procedures}

Details of previous episodes of $\mathrm{CM}$, current medications including antiretroviral therapy (ART) and antifungal medications, and self-reported adherence to medications were recorded. Reasons for patients not taking fluconazole secondary prophylaxis were ascertained by reviewing discharge scripts from the initial episode and by patient interviews, and categorised into patient non-adherence, or failure on the part of health care providers to provide the appropriate medication. In patients taking fluconazole prophylaxis, episodes were categorised into either IRIS or non-IRIS relapses, with IRIS defined as (i) microbiologically confirmed first episode of CM, (ii) resolution of CM symptoms before starting ART, (iii) self-reported adherence to fluconazole and ART, (iv) recurrence of CM symptoms after initiation of $\mathrm{ART}$, and $(v)$ no alternative diagnosis, including fluconazole resistance, found on laboratory testing and clinical review. All patients had a full clinical work-up including lumbar puncture, cell counts, protein and glucose quantification, bacterial cultures and TB cultures where appropriate, and fluconazole mean inhibitory concentrations (MICs) when fluconazole resistance was queried. CM-IRIS cases were further categorised as either CSF culture-negative or -positive.

During the last 12 months of the study, patients were also interviewed by a research nurse to determine their levels of knowledge about secondary fluconazole prophylaxis, and what education about the importance and correct usage of such prophylaxis they were given on discharge from hospital. Additional demographic, clinical and laboratory data, including HIV status, CD4 counts, CSF findings and fluconazole mean inhibitory concentrations (MICs), where available, were obtained from patient hospital notes and computerised laboratory records. In-hospital mortality was recorded for all patients. Fluconazole MICs were determined using Etests ( $\mathrm{AB}$ Biodisk). Plates were incubated at $35^{\circ} \mathrm{C}$ for 48 hours, and MICs were the lowest concentration at which the elliptical inhibition zone intercepted the scale on the strip. Etests have been shown to yield results in good agreement with the broth microdilution method. ${ }^{23}$ A definitive breakpoint MIC has yet to be established, and results were therefore interpreted on the best available evidence using the following criteria: susceptible - MIC $\leq 8 \mathrm{mg} / \mathrm{ml}$; dose-dependent susceptibility - MIC $16-32 \mathrm{mg} / \mathrm{ml}$; and resistant - MIC $\geq 64$ $\mathrm{mg} / \mathrm{ml}^{10,24-26}$

\begin{abstract}
Analysis
Data were analysed using Stata version 10 (StataCorp). Continuous variables were described as medians with interquartile ranges, and compared using the median test. Categorical data were described using proportions and compared using Pearson's $\chi^{2}$ test or Fisher's exact test. Statistical significance was defined as $p<0.05$. Odds ratios for mortality with $95 \%$ CIs were calculated using logistic regression analysis.
\end{abstract}

\section{Results}

\section{Patients}

There were 300 episodes of laboratory-confirmed CM; 69 were episodes of symptomatic relapse, occurring in 57 patients $(6$ patients had 2 episodes, 1 had 3, and 1 had 5), accounting for $23 \%$ of all laboratory-confirmed cases; $43 \%(N=30)$ of relapse episodes were in patients not taking fluconazole prophylaxis; $45 \%$ ( $N=31)$ were due to IRIS, and the remaining $12 \%(N=8)$ were relapses in patients taking fluconazole prophylaxis, but prior to starting ART (Fig. 1). Education of patients about the need for ongoing fluconazole prophylaxis was poor, with $38 \%$ (9) of 24 patients questioned (12 IRIS cases, 11 not taking fluconazole and 1 relapse on fluconazole prior to ART) reporting having received no information about the requirement for fluconazole secondary prophylaxis. There was a correlation between education and adherence to fluconazole. Of the 9 who received no education, 7 were non-adherent $(78 \%)$, versus 4 of the $15(27 \%)$ who were educated $(p=0.015)$.

Patient demographic, clinical and laboratory features of the relapse episodes are shown in Table I. Overall in-hospital mortality was $23 \%$. Factors significantly associated with inhospital mortality by univariate analysis were abnormal mental status (odds ratio (OR) 7.2, 95\% CI 2.1 - 25.0) and high fungal burden, evidenced by India ink-positivity (OR 5.0, 95\% CI 1.02 - 24.1).

\section{Inadequate secondary fluconazole prophylaxis}

Patients developing symptomatic relapse of $\mathrm{CM}$ due to inadequate fluconazole prophylaxis had severe disease, with a high proportion of abnormal mental status (30\%), high fungal 


\section{Original Articles}

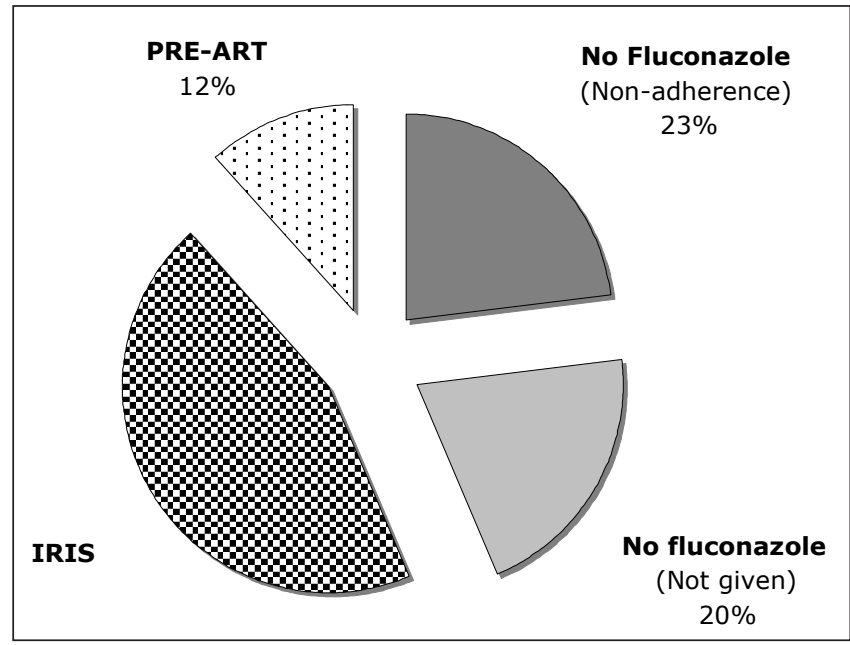

Fig. 1. Causes of symptomatic relapse of cryptococcal meningitis, divided into immune reconstitution inflammatory syndrome (IRIS) in patients taking ART, absence of fluconazole secondary prophylaxis (subdivided into patient non-adherence and not given-either not prescribed, not dispensed, or not continued at primary care level), and relapses in patients taking fluconazole prior to starting ART.

burdens (70\% India ink-positive) and high in-hospital mortality (33\%). Patients not taking fluconazole were 2.5 times more likely to die in hospital than those on appropriate fluconazole prophylaxis (OR, 95\% CI $0.8-8.0, p=0.11$, univariate analysis).

Of the 30 patients not taking fluconazole, 16 (53\%) had been prescribed fluconazole but were non-adherent to therapy. The remaining 14 (47\%) had either not been prescribed secondary prophylaxis on discharge from hospital after their initial episode of $\mathrm{CM}$, or had not been appropriately referred to primary care, or the prescription had not been continued by their primary care providers.

\section{IRIS}

Patients developing symptomatic relapse of CM due to IRIS had milder disease, with a lower proportion of abnormal mental status (19\%) and lower fungal burdens (55\% India ink-positive) than the other relapse cases, and low in-hospital mortality (13\%), although these differences did not reach statistical significance. IRIS episodes occurred a median of 61 days (IQR 31 - 146) after commencing ART. CSF cryptococcal cultures were positive in 12 (39\%) of the IRIS episodes. Fluconazole MICs were performed on 10 of these samples. None of the samples had an MIC $\geq 64 \mu \mathrm{g} / \mathrm{ml}$, suggesting fluconazole resistance. One had an MIC of $32 \mu \mathrm{g} / \mathrm{ml}$, indicating dose-dependent susceptibility, raising the possibility that decreased fluconazole susceptibility was a factor in this case, while the remaining 9 all had MICs $\leq 8 \mu \mathrm{g} / \mathrm{ml}$, indicating susceptibility to fluconazole. Although neither a documented immunological or virological response to ART, nor a decrease in fungal burden despite worsening symptoms were required for a diagnosis of IRIS, these were recorded in all but 2 patients. In each of the 19 of 31 IRIS cases where CD4 and viral load data were available, all showed a marked decrease in viral load, and rising CD4 counts. In terms of decreasing fungal burden, 19 of 31 cases were culture-negative, having been culture-positive during the initial episode. A further 4 of the culture-positive cases had a low fungal burden as evidenced by being India ink-negative at the relapse presentation, 2 more had very scanty growth at the relapse presentation, and 2 had a documented decrease in cryptococcal latex antigen test (CLAT) titre. Of the 2 patients who did not have either a documented immunological and viral response, or a documented decrease in fungal burden despite worsening symptoms, a diagnosis of IRIS was clinically made, after ruling out other potential diagnoses, including possible fluconazole resistance (both had fluconazole MICs indicating susceptibility, and were reportedly adherent to their medication).

\section{Relapses prior to ART}

Eight patients developed symptomatic relapse episodes while reporting adherence to appropriate secondary fluconazole prophylaxis, but before commencing ART. Of these, 4 were early relapses (within 1 month of discharge), and probably represent failure to respond to initial treatment rather than true relapse. The remaining 4 cases occurred at 50, 70, 104

Table I. Demographics, clinical features and outcomes of 69 episodes of symptomatic relapse of cryptococcal meningitis

\begin{tabular}{|c|c|c|c|c|}
\hline & IRIS $(N=31)$ & No fluconazole $(N=30)$ & Pre-ART, on fluconazole $(N=8)$ & $p$-value \\
\hline Age (years) & $33(29-42)^{+}$ & $35(28-42)$ & $33(26-39)$ & 0.63 \\
\hline Sex (\% male $)$ & $52 \%$ & $50 \%$ & $50 \%$ & 0.99 \\
\hline CD4 count $(\text { cells } / \mu \mathrm{l})^{\ddagger}$ & $77(15-130)$ & $46(20-154)$ & $60(39-109)$ & 0.84 \\
\hline $\begin{array}{l}\text { Time from initial episode to } \\
\text { relapse (days) }\end{array}$ & $87(56-146)$ & $103(67-156)$ & $48(23-96)$ & 0.28 \\
\hline On TB treatment & $37 \%(11)$ & $46 \%(12)$ & $25 \%(2)$ & 0.53 \\
\hline $\begin{array}{l}\text { Abnormal mental } \\
\text { status }(\%)^{\S}\end{array}$ & $19 \%(6)$ & $30 \%(9)$ & $25 \%(2)$ & 0.63 \\
\hline CSF lymphocytes $\left(x 10^{6} / 1\right)$ & $7(4-65)$ & $8(1-58)$ & $6(1-56)$ & 0.99 \\
\hline CSF protein $(\mathrm{g} / \mathrm{dl})$ & $1.08(0.8-1.6)$ & $0.97(0.5-2.0)$ & $0.4(0.3-0.8)$ & 0.07 \\
\hline CSF glucose $(\mathrm{mmol} / \mathrm{l})$ & $2.0(1.5-2.7)$ & $2.0(1.2-2.7)$ & $2.6(1.5-3.1)$ & 0.28 \\
\hline India ink- positive (\%) & $55 \%(17)$ & $70 \%(21)$ & $88 \%(7)$ & 0.17 \\
\hline In-hospital mortality & $13 \%(4)$ & $33 \%(10)$ & $25 \%(2)$ & 0.17 \\
\hline \multicolumn{5}{|c|}{ * $p$-values were calculated using Pearson's $\chi^{2}$-test or the median test. } \\
\hline \multicolumn{5}{|c|}{${ }^{\dagger}$ Continuous variables are expressed as median (inter-quartile range). } \\
\hline \multicolumn{5}{|c|}{${ }^{\ddagger} \mathrm{CD} 4$ cell count at time of relapse or most recent count within 6 months prior to relapse. } \\
\hline \multicolumn{5}{|c|}{$\S_{\text {Abnormal mental status was defined as any reduction in level of consciousness (confusion or Glasgow coma score }<15 \text { ) or a history of seizures. }}$} \\
\hline
\end{tabular}


and 196 days after the initial episode. Fluconazole MICs were performed in 2 of the 4 cases, and were both $\leq 8 \mu \mathrm{g} / \mathrm{ml}$. In 3 of these 8 cases, treatment of the initial episode of CM was inadequate (1 patient received fluconazole monotherapy, 2 had less than 2 weeks of amphotericin B). Two of the 3 patients who received inadequate initial therapy relapsed early (at 21 and 26 days after initial diagnosis), supporting the suggestion that these early relapses represented failure of initial treatment. The third patient relapsed at 50 days.

\section{Discussion}

Symptomatic relapse cases account for a large proportion (23\%) of all CM cases admitted to our hospital; $43 \%$ of these episodes occur in patients not taking secondary fluconazole prophylaxis, and whose outcomes are poor, with one-third dying in hospital. Much of this morbidity, mortality and the associated costs to the health service are entirely preventable, as evidence for the efficacy of fluconazole consolidation and maintenance therapy is strong, ${ }^{16,17,27}$ treatment guidelines are clear, ${ }^{18}$ and the drug is made freely available to the South African public health care sector. That so many patients are presenting with symptomatic relapses while not taking fluconazole demonstrates a failure of the health care services, and highlights inadequacies in patient education, discharge planning and communication between secondary and primary providers. In $47 \%$ of these cases, fluconazole had not been prescribed or dispensed by health care providers, or patients had not been properly referred to primary care services for continuation of their fluconazole. In the remaining cases, patient non-adherence, in part, reflects a lack of patient education surrounding the importance of secondary prophylaxis $-38 \%$ of patients questioned reported having received no relevant education. Factors probably contributing to the inadequate patient education, and poor prescription, dispensing of, and referral for fluconazole in our setting, in common with many public sector health care facilities in South Africa, are heavy workloads, high turnover of junior staff, and language barriers.

Even with appropriate secondary fluconazole prophylaxis, not all cases of symptomatic relapse can be prevented; $45 \%$ of relapses in this study were due to IRIS, and $12 \%$ occurred in patients prior to ART, despite taking fluconazole. Cryptococcal IRIS is well recognised and occurs in $6-30 \%$ of patients with cryptococcal meningitis following commencement of ART.,2, IRIS accounted for 10\% (31 of 300) of all our CM episodes, occurring at a median of 61 days after commencing ART, consistent with 3 South African cohorts where the median time to onset of IRIS symptoms after starting ART was 1 - 2 months. ${ }^{10,12,30}$ Compared with the non-IRIS relapse cases in this cohort, the patients presenting with IRIS had less severe clinical disease, lower fungal burdens and a lower mortality of 13\%.

There were few cases of symptomatic relapse in this cohort occurring in patients taking fluconazole and prior to commencing ART. Once very early relapses (within 1 month), which probably represented ongoing manifestations of the initial presentation, were excluded, there were only 4 patients in this category. These cases may represent true failures of fluconazole prophylaxis, although poor fluconazole adherence is a possibility, as we only assessed adherence by self-reporting.
In the 2 cases without MICs available, fluconazole resistance cannot be excluded as a factor.

Of note was that no fluconazole-resistant isolates of $C$. neoformans (MIC $\geq 64 \mu \mathrm{g} / \mathrm{ml}$ ) were seen during the entire 2-year study period, contrasting markedly with an earlier study involving patients from the same hospital. ${ }^{10}$ Between 2003 and 2005, after patients not taking fluconazole secondary prophylaxis were excluded, 32 relapse episodes in 27 patients were reported. Of these, 21 were culture-positive, of whom 14 had isolates with resistance to fluconazole (MIC $\geq 64 \mu \mathrm{g} / \mathrm{ml}$ ), and a further 2 had isolates with dose-dependent susceptibility (MIC $16-32 \mu \mathrm{g} / \mathrm{ml}$ ). ${ }^{10}$ An explanation for this disparity is the switch to amphotericin B as initial treatment at our hospital, in early 2005. At the time of the 2003 - 2005 study, the standard initial therapy for CM was fluconazole monotherapy $400 \mathrm{mg}$ / day. Clearance of cryptococci from CSF is very slow with fluconazole, ${ }^{4}$ a fungistatic agent, and the use of fluconazole as initial therapy might have promoted drug resistance development owing to ongoing high fungal burdens and drug exposure over prolonged periods. ${ }^{31}$ The fungicidal effects of amphotericin $\mathrm{B}$, leading to faster clearance of cryptococci from the $\mathrm{CSF}^{4}$ followed by switching to fluconazole once the fungal burden had been markedly reduced, appears to have minimised the risk of developing later fluconazole resistance.

Our study had several limitations, notably the reliance on patient self-reporting of both fluconazole adherence, and of education received relating to secondary prophylaxis on discharge from hospital after the initial meningitis episode. While the number of patients developing relapse prior to ART while reporting adherence to fluconazole was small, and false reporting of non-adherence is unlikely, non-adherence to fluconazole cannot be entirely ruled out as a factor in some of the culture-positive IRIS cases.

In conclusion: relapses of previously treated disease constitute a significant proportion of the $\mathrm{CM}$ disease burden. A switch from fluconazole to amphotericin $B$ as initial treatment has been associated with virtual elimination of the problem of secondary fluconazole resistance at our centre. However, large numbers of relapses are due to inadequate use of secondary fluconazole prophylaxis, and are entirely preventable. Efforts must be made to strengthen both patient and provider knowledge about the importance of secondary fluconazole prophylaxis in treatment of $\mathrm{CM}$ and to improve health system performance in this regard.

Joseph Jarvis and Graeme Meintjes received support from the Wellcome Trust, London, UK.

\footnotetext{
References

1. Hakim JG, Gangaidzo IT, Heyderman RS, et al. Impact of HIV infection on meningitis in Harare, Zimbabwe: a prospective study of 406 predominantly adult patients. AIDS 2000; 14(10): 1401-1407.

2. Scarborough $\mathrm{M}$, Gordon $\mathrm{SB}$, Whitty $\mathrm{CJ}$, et al. Corticosteroids for bacterial meningitis in adults in sub-Saharan Africa. N Engl J Med 2007; 357(24): 2441-2450.

3. Harling G, Orrell C, Wood R. Healthcare utilization of patients accessing an African national treatment program. BMC Health Serv Res 2007; 7: 80.

4. Bicanic T, Meintjes G, Wood R, et al. Fungal burden, early fungicidal activity, and outcome in cryptococcal meningitis in antiretroviral-naive or antiretroviral-experienced patients treated with amphotericin B or fluconazole. Clin Infect Dis 2007; 45(1): 76-80.

5. Kambugu A, Meya DB, Rhein J, et al. Outcomes of cryptococcal meningitis in Uganda before Kambugu A, Meya DB, Rhein J, et al. Outcomes of cryptococcal meningitis in Uganda befor
and after the availability of highly active antiretroviral therapy. Clin Infect Dis 2008; 46(11): 1694-1701.
} 


\section{Original Articles}

6. Okongo M, Morgan D, Mayanja B, Ross A, Whitworth J. Causes of death in a rural, population-based human immunodeficiency virus type 1 (HIV-1) natural history cohort in Uganda. Int J Epidemiol 1998; 27(4): 698-702.

7. French N, Gray K, Watera C, et al. Cryptococcal infection in a cohort of HIV-1-infected Ugandan adults. AIDS 2002; 16(7): 1031-1038.

8. Corbett EL, Churchyard GJ, Charalambos S, et al. Morbidity and mortality in South African gold miners: impact of untreated disease due to human immunodeficiency virus. Clin Infect Dis 2002; 34(9): 1251-1258

9. Park BJ, Wannemuehler KA, Marston BJ, Govender N, Pappas PG, Chiller TM. Estimation of the current global burden of cryptococcal meningitis among persons living with HIV/AIDS AIDS 2009; 23(4): 525-530.

10. Bicanic T, Harrison T, Niepieklo A, Dyakopu N, Meintjes G. Symptomatic relapse of HIV-associated cryptococcal meningitis after initial fluconazole monotherapy: the role of

11. Lawn SD, Bekker LG, Myer L, Orrell C, Wood R. Cryptococcocal immune reconstitution disease: a major cause of early mortality in a South African antiretroviral programme. AIDS

12. Bicanic $\mathrm{T}$, Meintjes $\mathrm{G}$, Rebe $\mathrm{K}$, et al. Immune reconstitution inflammatory syndrome in HIVassociated cryptococcal meningitis: A prospective study. J Acquir Immune Defic Syndr 2009, 51: 130 .

13. Zuger A, Louie E, Holzman RS, Simberkoff MS, Rahal JJ. Cryptococcal disease in patients with the acquired immunodeficiency syndrome. Diagnostic features and outcome of treatment. Ann Intern Med 1986; 104(2): 234-240.

14. Kovacs JA, Kovacs AA, Polis M, et al. Cryptococcosis in the acquired immunodeficiency syndrome. Ann Intern Med 1985; 103(4): 533-538.

15. Chuck SL, Sande MA. Infections with Cryptococcus neoformans in the acquired immunodeficiency syndrome. N Engl J Med 1989; 321(12): 794-799.

16. Bozzette SA, Larsen RA, Chiu J, et al. A placebo-controlled trial of maintenance therapy with fluconazole after treatment of cryptococcal meningitis in the acquired immunodeficiency syndrome. California Collaborative Treatment Group. N Engl J Med 1991; 324(9): 580-584.

17. Powderly WG, Saag MS, Cloud GA, et al. A controlled trial of fluconazole or amphotericin B to prevent relapse of cryptococcal meningitis in patients with the acquired immunodeficiency syndrome. The NIAID AIDS Clinical Trials Group and Mycoses Study Group. N Engl J Med 1992; 326(12): 793-798.

18. Guidelines for the prevention, diagnosis and management of cryptococcal meningitis and disseminated cryptococcosis in HIV-infected patients. Southern African Journal of HIV Medicine 2007; 28: 25-35.

19. Collett G, Parrish A. Fluconazole donation and outcomes assessment in cryptococcal meningitis. S Afr Med I 2007; 97(3): 175-176.
20. Western Cape HIV Antenatal Sero-prevalence Survey in South Africa: 2007. Pretoria: Department of Health, 2007.

21. Jarvis JN, Williams A, Crede T, Harrison TS, Meintjes G. Adult meningitis in a setting of high HIV and TB prevalence: Findings from 4961 cases. 5th IAS Conference on HIV Pathogenesis, Treatment and Prevention, Cape Town 2009; 19 - 22 July 2009 (Abstract no. 2915).

22. Jarvis JN, Boulle A, Loyse A, et al. High ongoing burden of cryptococcal disease in Africa despite antiretroviral roll out. AIDS 2009, 23: 1181-1185.

23. Pfaller MA, Messer SA, Karlsson A, Bolmstrom A. Evaluation of the Etest method for determining fluconazole susceptibilities of 402 clinical yeast isolates by using three different agar media. J Clin Microbiol 1998; 36(9): 2586-2589.

24. NCCLS. Minutes NCCLS antifungal susceptibility subcommittee meeting on interpretive breakpoints.Villanova, PA: NCCLS, 1996.

25. Aller AI, Martin-Mazuelos E, Lozano F, et al. Correlation of fluconazole MICs with clinical outcome in cryptococcal infection. Antimicrob Agents Chemother 2000; 44(6): 1544-1548.

26. Witt MD, Lewis RJ, Larsen RA, et al. Identification of patients with acute AIDS-associated cryptococcal meningitis who can be effectively treated with fluconazole: the role of antifungal susceptibility testing. Clin Infect Dis 1996; 22(2): 322-328.

27. Van der Horst CM, Saag MS, Cloud GA, et al. Treatment of cryptococcal meningitis associated with the acquired immunodeficiency syndrome. National Institute of Allergy and Infectious Diseases Mycoses Study Group and AIDS Clinical Trials Group. N Engl J Med 1997; 337(1): 15-21.

28. Shelburne SA, Darcourt J, White AC jun., et al. The role of immune reconstitution inflammatory syndrome in AIDS-related Cryptococcus neoformans disease in the era of highly active antiretroviral therapy. Clin Infect Dis 2005; 40(7): 1049-1052.

29. Lortholary O, Fontanet A, Memain N, Martin A, Sitbon K, Dromer F. Incidence and risk factors of immune reconstitution inflammatory syndrome complicating HIV-associated cryptococcosis in France. AIDS 2005; 19(10): 1043-1049.

30. Murdoch DM, Venter WD, Feldman C, Van Rie A. Incidence and risk factors for the immune reconstitution inflammatory syndrome in HIV patients in South Africa: a prospective study. AIDS 2008; 22(5): 601-610.

31. Jarvis JN, Bicanic T, Harrison TS. Treatment of HIV-associated cryptococcal meningitis in South Africa: the case for amphotericin B over conventional dose fluconazole for initial therapy Southern African Journal of HIV Medicine 2007; 28: 36-39.

Accepted 26 August 2009. 